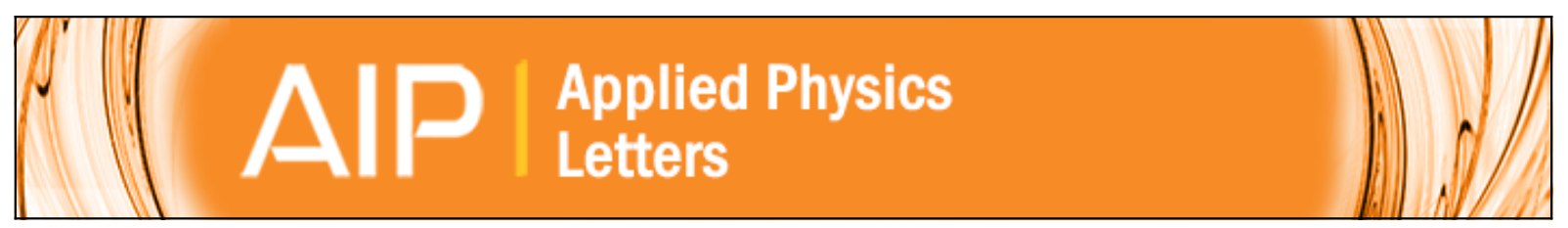

\title{
Metal/graphene sheets as p-type transparent conducting electrodes in GaN light emitting diodes
}

Jung Min Lee, Hae Yong Jeong, Kyoung Jin Choi, and Won II Park

Citation: Applied Physics Letters 99, 041115 (2011); doi: 10.1063/1.3595941

View online: http://dx.doi.org/10.1063/1.3595941

View Table of Contents: http://scitation.aip.org/content/aip/journal/apl/99/4?ver=pdfcov

Published by the AIP Publishing

\section{Articles you may be interested in}

Enhancement of light output power in ultraviolet light emitting diodes using graphene film on self-assembled Au nanocluster by agglomeration process

J. Appl. Phys. 114, 223105 (2013); 10.1063/1.4836835

Graphene-silver nanowire hybrid structure as a transparent and current spreading electrode in ultraviolet light emitting diodes

Appl. Phys. Lett. 103, 051105 (2013); 10.1063/1.4817256

Three-dimensional graphene foam-based transparent conductive electrodes in GaN-based blue light-emitting diodes

Appl. Phys. Lett. 102, 161902 (2013); 10.1063/1.4801763

Partially sandwiched graphene as transparent conductive layer for InGaN-based vertical light emitting diodes Appl. Phys. Lett. 101, 061102 (2012); 10.1063/1.4742892

Graphene network on indium tin oxide nanodot nodes for transparent and current spreading electrode in InGaN/GaN light emitting diode

Appl. Phys. Lett. 98, 251114 (2011); 10.1063/1.3601462

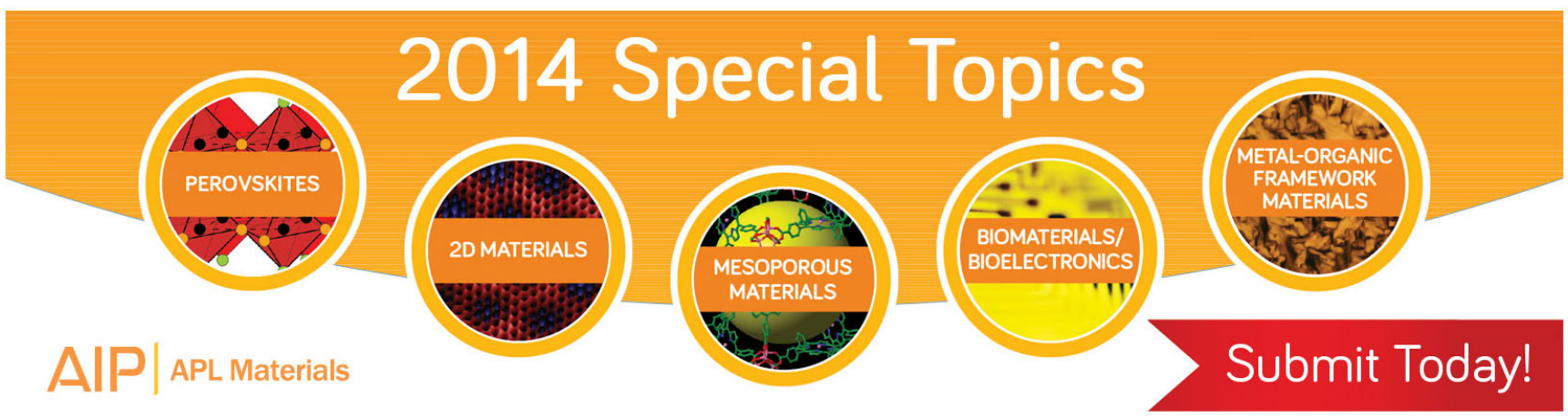




\title{
Metal/graphene sheets as p-type transparent conducting electrodes in GaN light emitting diodes
}

\author{
Jung Min Lee, ${ }^{1}$ Hae Yong Jeong, ${ }^{1}$ Kyoung Jin Choi, ${ }^{2}$ and Won II Park ${ }^{1, a)}$ \\ ${ }^{1}$ Division of Materials Science Engineering, Hanyang University, Seoul 133-791, Korea \\ ${ }^{2}$ Nano-Photonics Center, Korea Institute of Science and Technology, Seoul, 130-650, Korea
}

(Received 12 April 2011; accepted 9 May 2011; published online 29 July 2011)

\begin{abstract}
We demonstrate the use of graphene based transparent sheets as a p-type current spreading layer in GaN light emitting diodes (LEDs). Very thin Ni/Au was inserted between graphene and p-type GaN to reduce contact resistance, which reduced contact resistance from $\sim 5.5$ to $\sim 0.6 \Omega / \mathrm{cm}^{2}$, with no critical optical loss. As a result, LEDs with metal-graphene provided current spreading and injection into the p-type GaN layer, enabling three times enhanced electroluminescent intensity compared with those with graphene alone. We confirmed very strong blue light emission in a large area of the metal-graphene layer by analyzing image brightness. (c) 2011 American Institute of Physics. [doi:10.1063/1.3595941]
\end{abstract}

Low resistance, transparent Ohmic contact to p-type $\mathrm{GaN}$ is crucial to improve current injection and light extraction efficiency for the realization of solid-state lighting using GaN-based blue light emitting diodes (LEDs). ${ }^{1} \mathrm{Ni}$-, Au- or Pt-based metal electrodes have been used to reduce contact resistance. ${ }^{2,3}$ However, due to the difficulty in achieving heavily doped p-type GaN, current spreading over a large area cannot be achieved by the use of these opaque metal electrodes. Alternatively, tin-doped indium oxide (ITO) has been employed as a transparent conducting electrode for $\mathrm{p}$ type GaN. ${ }^{4}$ Despite the improvement in device performance with ITO-based transparent p-type contacts, the exclusive use of these oxides becomes increasingly problematic due to the cost and dwindling supply of indium, chemical instability in the presence of acids or bases, and low optical transmittance in the ultraviolet and blue wavelength regions. ${ }^{4}$

Recently, ultrathin graphene sheets have attracted great attention as alternative transparent conducting electrodes to ITO due to their excellent conductivity, good transparency, and chemical stability. However, the use of bare graphene as a window electrode in p-type $\mathrm{GaN}$ poses intrinsic limitations for current injection due to its work function $(\sim 4.5 \mathrm{eV})$, which is smaller than p-type $\mathrm{GaN}(\sim 7.5 \mathrm{eV}) .^{5}$ Herein, our approach to overcome this problem involves inserting very thin metal layers between graphene and p-type GaN; the graphene in this case provides efficient current spreading while thin metal layers lead to improved contact properties without significant optical loss. The resulting GaN-based blue LEDs showed high performance with strong blue light emission over a large area of the metal/graphene (M/Gr) layer.

Figure 1 shows schematic diagrams of GaN-based LEDs fabricated using three types of p-type electrodes: Ni/Au finger stripes (Type I), finger stripes covered with graphene (Type II), and finger stripes deposited with a thin metal layer and graphene (Type III). GaN-based epitaxial layers consisting of undoped GaN, Si-doped n-GaN, InGaN/GaN multiple-quantum-wells (MQWs), and $\mathrm{Mg}$-doped p-GaN were grown on sapphire (0001) substrates by metal-organic chem-

${ }^{a)}$ Electronic mail: wipark@hanyang.ac.kr. ical vapor deposition (MOCVD). After chemical etching with aqua regia, $\mathrm{Ni} / \mathrm{Au}(10 \mathrm{~nm} / 30 \mathrm{~nm})$ finger stripes were fabricated on p-type GaN layers by photolithography and metal evaporation. The line width and spacing of the stripes were $80 \mu \mathrm{m}$ and $2500 \mu \mathrm{m}$, respectively. To make n-type Ohmic contact electrodes, the GaN epilayers were etched via an inductively coupled plasma process with $\mathrm{BCl}_{3} / \mathrm{Cl}_{2}$ source gases until n-type GaN layers were exposed. This was followed by deposition of Ti/Al $(10 \mathrm{~nm} / 30 \mathrm{~nm})$ layers. After fabricating these samples (Type I), fabrication of the Type II device was completed by transferring a single layer of graphene onto the $\mathrm{Ni}$ /Au stripes. In parallel, a Type III device was also achieved by inserting very thin metal $(\mathrm{Ni}$ or $\mathrm{Ni} / \mathrm{Au}$ ) layer between the graphene and p-type GaN layer. In this way, single-layer graphene films were grown on copper foils $(25 \mu \mathrm{m}$ thick) by $\mathrm{CVD}$ at $1000^{\circ} \mathrm{C}$ for $30 \mathrm{~min}$ under methane [35 SCCM (SCCM denotes cubic centimeter per minute at STP)] and $\mathrm{H}_{2}$ [2 SCCM] flow. ${ }^{6}$ Graphene sheet resistance was measured to be in the range of $100-200 \Omega$ /square. As-grown graphene sheets were capped with poly(methyl methacrylate) (PMMA) and were detached from the copper foils with diluted ammonium persulfate acid before being transferred to the target device surfaces. After removing the PMMA support with acetone, all devices were annealed at $300{ }^{\circ} \mathrm{C}$ for 1 min under a nitrogen ambient in a rapid thermal annealing system.

To evaluate the quality and number of graphene layers, Raman spectroscopy was performed with an

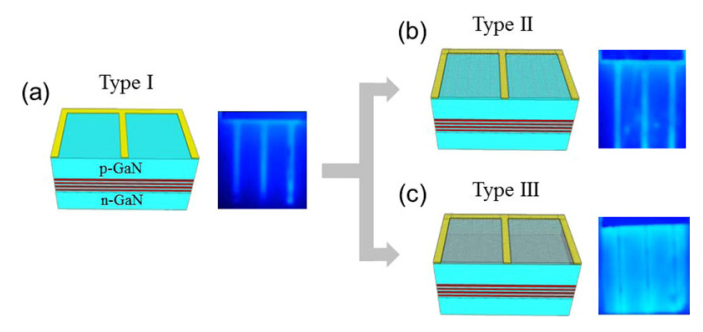

FIG. 1. (Color online) Schematic illustrations and photographs of GaN-based LEDs consisting of three types of p-type electrodes: (a) LED with Ni/Au finger stripe electrodes (Type I), (b) finger stripes with graphene (Type II), and (c) finger stripes deposited with thin metal layer and graphene (Type III). 

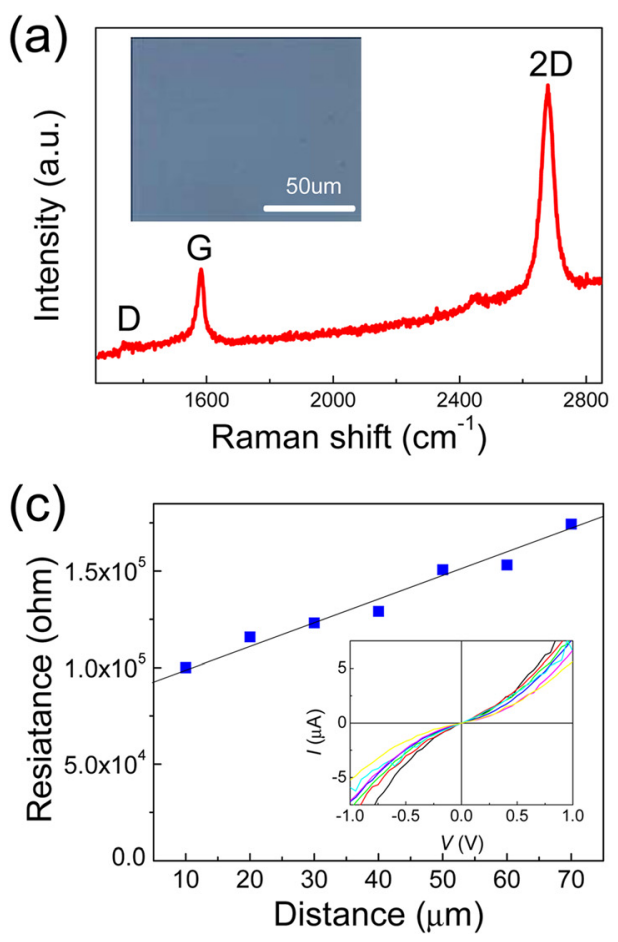
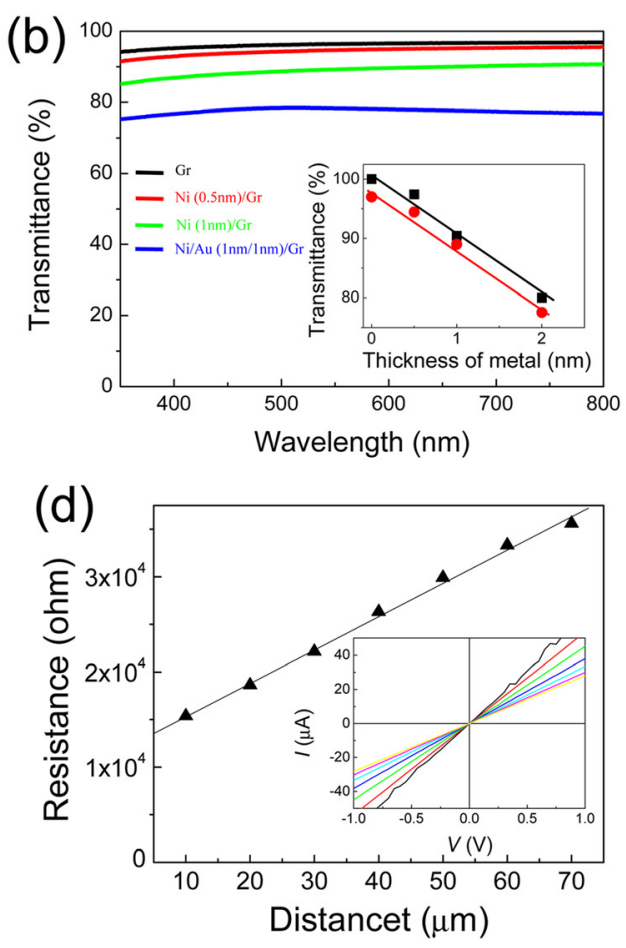

FIG. 2. (Color online) (a) Representative Raman spectra and optical image (inset) of single-layer graphene. (b) Optical transmittance spectra of graphene, $0.5 \mathrm{~nm} \mathrm{Ni} /$ graphene, $1 \mathrm{~nm} \mathrm{Ni/graphene,}$

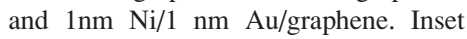
transmittance of metal (squares) and metal/graphene (circles) layers as a function of metal thickness. (c) and (d) Resistance as a function of the spacing between the square $(100 \mu \mathrm{m} \times 100 \mu \mathrm{m})$ pads of graphene (c) and $\mathrm{Ni} / \mathrm{Au} /$ graphene (d) on the p-GaN layer. Insets show the I-V characteristic curves measured for different pad spacings. excitation wavelength of $514 \mathrm{~nm}$. Figure 2(a) shows clear G $\left(\sim 1582 \mathrm{~cm}^{-1}\right)$ and two-dimensional (2D) bands $\left(\sim 2678 \mathrm{~cm}^{-1}\right)$ with an extremely small disorder-induced D band $\left(\sim 1360 \mathrm{~cm}^{-1}\right)$, demonstrating the high structural quality of the graphene films. The graphene was verified to be predominantly single-layer by a higher $2 \mathrm{D}$ peak intensity than that of the $\mathrm{G}$ band with a $2 \mathrm{D}-$ to- $\mathrm{G}$ intensity ratio $\left(\mathrm{I}_{2 \mathrm{D}} / \mathrm{I}_{\mathrm{G}}\right)$ of $\sim 2$, and a single Lorentzian profile of the $2 \mathrm{D}$ band with a narrow full width at half-maximum of $\sim 41 \mathrm{~cm}^{-1}$. $^{7}$ Indeed, the optical absorption by graphene is below $2.5 \%$ [black line in Fig. 2(b)], which is quite close to theoretical value for a graphene monolayer $\left[\pi\left(e^{2} / \hbar c\right) \approx 2.3 \%\right]^{8}$

We also investigated the decrease in optical transmittance by inserting a thin metal layer below graphene [Fig. 2(b)]. Indeed, transmittance of visible light monotonously decreases with increasing metal layer thickness. For both metal and metal/graphene layers, the average transmittance shows a linear dependence on metal thickness with a negative slope of $(\sim 10 \% / \mathrm{nm})$, and metal thickness is more critical than graphene in optical loss. However, the transmittance of $\mathrm{M} / \mathrm{Gr}$ is still $\sim 90 \%$ for $\mathrm{Ni}(0.5 \mathrm{~nm}) /$ graphene and $\sim 78 \%$ for $\mathrm{Ni} / \mathrm{Au}(1 \mathrm{~nm} / 1 \mathrm{~nm}) / g r a p h e n e$, which suggests the potential use of graphene-based materials as window electrodes in LEDs.

While the optical transmittance is critical to efficient light extraction, the contact resistance is also important to improve the device performance (in terms of current injection without current crowding). ${ }^{9}$ The specific contact resistances of graphene and metal/graphene to p-type GaN were determined using a transmission line model (TLM) measurement [Figs. 2(c) and 2(d)]. To fabricate the TLM test structures, graphene and metal/graphene layers were deposited onto chemically etched p-type GaN, thermally annealed at $300{ }^{\circ} \mathrm{C}$ for $1 \mathrm{~min}$, and deposited with square metal pads
$(100 \mu \mathrm{m} \times 100 \mu \mathrm{m})$ with variable space between the pads. Finally, by using the square metal pads as an etching mask, $\mathrm{O}_{2}$ plasma etching $(30 \mathrm{~W}, 60 \mathrm{~s})$ produced TLM structures consisting of square pads of graphene [Fig. 2(c)] and metal/ graphene [Fig. 2(d)] on p-GaN. The current-voltage (I-V) characteristic curves of graphene contacts on $\mathrm{p}-\mathrm{GaN}$ are nonlinear and a contact resistance of $\sim 5.5 \Omega / \mathrm{cm}^{-2}$ is deduced from the intercept of the plot of resistance versus distance. This value is significantly higher than those of conventional metal contacts and is still higher than that of ITO contact to p-GaN. ${ }^{3}$ However, by inserting a thin Ni/Au $(1 \mathrm{~nm} / 1 \mathrm{~nm})$, we achieved a linear I-V characteristic behavior with a low sheet resistance below $0.6 \Omega / \mathrm{cm}^{-2}$ [Fig. 2(d)]. This result indicates that, despite the optical loss due to the metal, the hybrid of metal and graphene is more suitable for transparent Ohmic contact to $\mathrm{p}-\mathrm{GaN}$ than graphene alone.

Figure 3 compares the device characteristics of $\mathrm{GaN}$ LEDs consisting of three different kinds of p-contact electrodes (which correspond to Type I, II, and III devices in Fig. 1). Several key features are noted. First, all devices exhibited clear rectifying $\mathrm{I}-\mathrm{V}$ behaviors with a turn-on voltage of approximately $2.5 \mathrm{~V}$. Small reverse bias leakage currents (below $\sim 1 \mu \mathrm{A}$ ) were occasionally observed, which might be caused by unintentional defects in the GaN epilayers or in the interfacial regions. ${ }^{10}$ However, taking into account that the leakage current increases markedly with increasing electrode size, the small leakage current in our large devices $(0.6 \mathrm{~cm} \times 0.8 \mathrm{~cm})$ is promising. Second, when a voltage larger than the turn-on voltage is applied, an abrupt increase in current and the onset of light emission were observed. The current and electroluminescent (EL) intensity of Type II LEDs (graphene sheet on metal stripes) measured with a forward voltage of $3 \mathrm{~V}$ is close to those of the Type I LED (metal stripes). On the other hand, Type III LEDs consisting of $\mathrm{Ni} / \mathrm{Au} / \mathrm{graphene} \mathrm{p}$-contact electrodes showed enhanced 

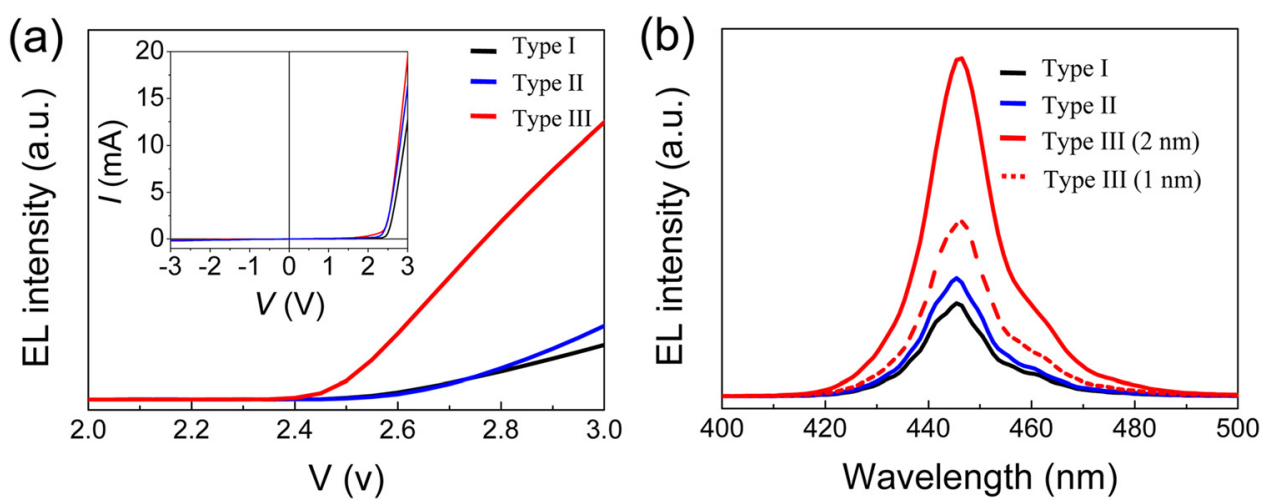

FIG. 3. (Color online) (a) EL intensity and current (inset) vs voltage curves for GaN LEDs. performance in terms of current injection and light emission, which is consistent with the result of contact resistance measurements [Figs. 2(c) and 2(d)]. It is also noted that, even in Type III LEDs, luminescence intensity is affected by metal thickness. To a certain extent, increase in metal thickness enhanced contact but involves optical loss. ${ }^{11}$ As such, there is room for significant improvement by finding the optimal thickness of intermediate metal layers. Third, despite the differences in EL intensity, all samples exhibited similar EL spectra with a dominant emission peak at $446 \mathrm{~nm}$, and no noticeable deep level emissions were observed [Fig. 4(c)].

Interestingly, EL intensity of Type III LED measured at $3 \mathrm{~V}$ was approximately four times stronger than those of Type I and II LEDs, although the current for Type III LEDs was increased by only $\sim 25 \%-65 \%$ relative to Type I and II LEDs. Such an observation agrees well with the photographs shown in Fig. 1 and reflects the different current spreading and injection behaviors among the three types of p-contact electrodes. To clarify this point, we more thoroughly investigated the luminescence profiles across two adjacent metal stripes of the LEDs. Micrograph of optical emission from Type I LED and a corresponding brightness profile [Fig. 4(a)] revealed an abrupt (exponential) decrease in the emission intensity with distance from the metal stripe edges, which represents the inevitable current crowding under the opaque metal stripes. The introduction of graphene slightly improved the current spreading characteristic but a large amount of contact resistance at the graphene and p$\mathrm{GaN}$ interface still prevented uniform current injection over

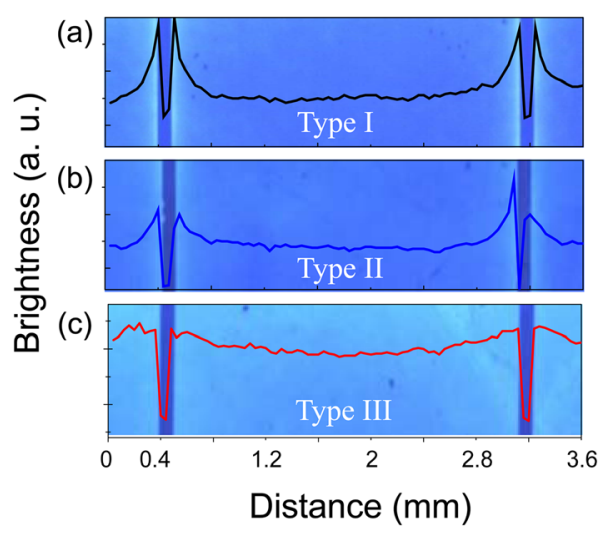

FIG. 4. (Color online) Micrographs of optical emission from three types of GaN LEDs, overlapped with brightness profiles across two adjacent finger stripes. the large area [Fig. 4(b)]. On the other hand, in the Type III LED, we observed clear blue light emission over the large area with a relatively uniform EL intensity profile [Fig. 4(c)]. Finally, we have also fabricated GaN LED by using thin $\mathrm{Ni} / \mathrm{Au}(1 \mathrm{~nm} / 1 \mathrm{~nm})$ layer as a window electrode to test current spreading characteristic of the thin metal layer. In this device, the introduction of thin metal layer rather resulted in $\sim 30 \%$ loss of EL intensity compared with Type I LED. Since this ultrathin metal layer might form threedimensional metal islands rather than $2 \mathrm{D}$ continuous film, it became highly resistive.

In conclusion, we fabricated GaN LEDs with very thin metal/graphene electrodes as a transparent and current injection layer in p-type GaN. By inserting a very thin metal $(\mathrm{Ni} /$ $\mathrm{Au})$ layer between monolayer graphene and p-type $\mathrm{GaN}$, contact resistance was reduced from 5.5 to $0.6 \Omega / \mathrm{cm}^{-2}$ while optical transmittance exceeded $\sim 78 \%$ for visible light. Due to the improvement in current spreading and injection characteristics, the GaN LEDs incorporated with metal/graphene electrodes demonstrated uniform blue light emission over a large area $(0.6 \mathrm{~cm} \times 0.8 \mathrm{~cm})$.

This research was supported by Future-based Technology Development Program (Nano Fields) through the National Research Foundation of Korea (NRF) funded by the Ministry of Education, Science and Technology (Grant No. 2010-0029300) and by the International Research \& Development Program of the NRF funded by the MEST (grant number K21002001635-10E0100-06310).

${ }^{1}$ F. A. Ponce and D. P. Bour, Nature (London) 386, 351 (1997).

${ }^{2}$ J. K. Sheu, Y. K. Su, G. C. Chi, P. L. Koh, M. J. Jou, C. M. Chang, C. C. Liu, and W. C. Hung, Appl. Phys. Lett. 74, 2340 (1999).

${ }^{3}$ D. W. Kim, Y. J. Sung, J. W. Park, and G. Y. Yeom, Thin Solid Films 398-399, 87 (2001).

${ }^{4}$ X. Wang, L. Zhi, and K. Mullen, Nano Lett. 8, 323 (2008).

${ }^{5}$ G. Jo, M. Choe, C.-Y. Cho1, J. H. Kim, W. Park, S. Lee, W.-K. Hong, T.-W. Kim, S.-J. Park, B. H. Hong, Y. H. Kahng, and T. Lee, Nanotechnology 21, 175201 (2010).

${ }^{6}$ X. Li, W. Cai, J. An, S. Kim, J. Nah, D. Yang, R. Piner, A. Velamakanni, I. Jung, E. Tutuc, S. K. Banerjee, L. Colombo, and R. S. Ruoff, Science 324, 1312 (2009).

${ }^{7}$ Z. Ni, Y. Wang, T. Yu, and Z. Shen, Nano Res. 1, 273 (2008).

${ }^{8}$ R. R. Nair, P. Blake, A. N. Grigorenko, K. S. Novoselov, T. J. Booth, T. Stauber, N. M. R. Peres, and A. K. Geim, Science 320, 1308 (2008).

${ }^{9}$ V. K. Malyutenko, S. S. Bolgov, and A. D. Podoltsev, Appl. Phys. Lett. 97, 251110 (2010).

${ }^{10}$ J. W. P. Hsu, M. J. Manfra, D. V. Lang, S. Richter, S. N. G. Chu, A. M. Sergent, R. N. Kleiman, and L. N. Pfeiffer, Appl. Phys. Lett. 78, 1685 (2001).

${ }^{11}$ Y. C. Lin, S. J. Chang, Y. K. Su, T. Y. Tsai, C. S. Chang, S. C. Shei, C. W. Kuo, and S. C. Chen, Solid-State Electron. 47, 849 (2003). 